\title{
Mesh-Based Transperineal Repair of a Perineal Hernia After a Laparoscopic Abdominoperineal Resection
}

\author{
Taek-Gu Lee, Sang-Jeon Lee \\ Department of Surgery, Chungbuk National University College of Medicine, Cheongju, Korea
}

\begin{abstract}
A perineal hernia $(\mathrm{PH})$ is formed by a protrusion of intra-abdominal viscera through a defect in the pelvic floor. This is a rare complication after a conventional abdominoperineal resection (APR). However, the risk of a PH may be increased after a laparoscopic resection because this technique can cause fewer postoperative adhesions, predisposing the small bowel to sliding down toward the pelvis. However, only a few case reports describe the transperineal approach for the repair of a $\mathrm{PH}$ after a laparoscopic APR. We present a case of a PH after a laparoscopic APR; the PH was repaired with synthetic mesh by using a transperineal approach. A transperineal approach using a mesh to reconstruct the pelvic floor is less invasive and more effective. We suggest that this technique should probably be the first choice for treating an uncomplicated $\mathrm{PH}$ that occurs after a laparoscopic APR.
\end{abstract}

Keywords: Perineal hernia; Laparoscopic abdominoperineal resection; Synthetic mesh; Transperineal approach

\section{INTRODUCTION}

A perineal hernia $(\mathrm{PH})$ is an incisional hernia caused by a protrusion into the perineum of intra-abdominal contents through a defect in the pelvic floor. This rare complication can occur after an abdominoperineal resection (APR) or a pelvic exenteration, sacrectomy or coccygectomy. Many postoperative $\mathrm{PHs}$ remain asymptomatic and undiscovered. Symptoms range from bulging with discomfort in the perineum to urinary problems, small bowel obstruction and skin erosion. Clinical diagnosis may be difficult as they are usually asymptomatic, and a high index of suspicion is required in patients with perineal discomfort, even in the absence of a bulging mass. During examination, the intestinal nature of the hernia sac can be confirmed by the peristaltic sounds associated with perineal protrusion. The incidence of clinically-significant

Received: February 12, 2014 - Accepted: March 18, 2014

Correspondence to: Sang-Jeon Lee, M.D.

Department of Surgery, Chungbuk National University College of Medicine, 410 Sunbong-ro, Heungdeok-gu, Cheongju 361-763, Korea

Tel: +82-43-269-6360, Fax: +82-43-266-6037

E-mail: colon@chungbuk.ac.kr

(C) 2014 The Korean Society of Coloproctology

This is an open-access article distributed under the terms of the Creative Commons Attribution NonCommercial License (http://creativecommons.org/licenses/by-nc/3.0) which permits unrestricted noncommercial use, distribution, and reproduction in any medium, provided the original work is properly cited.
PHs is supposed to be $<1 \%$ after a conventional APR. However, its incidence after a laparoscopic APR remains unknown at present. Only a few case reports in the literature describe the transperineal approach for the repair of a postoperative hernia with synthetic material after a laparoscopic APR $[1,2]$. We present a case of a PH after a laparoscopic APR; the hernia was repaired with synthetic mesh by using a transperineal approach.

\section{CASE REPORT}

A 73-year-old man underwent a laparoscopic APR with curative intent for rectal cancer in an outside hospital. There were no complications during the postoperative period. Pathologic examination revealed a well-differentiated adenocarcinoma, and the tumor stage was IIIB (pT3N1aM0). There was no lymphovascular invasion or perineural invasion. He received postoperative adjuvant chemoradiotherapy consisting of capecitabine with 45 Gy in 25 fractions with a 5.4-Gy boost. The patient complained of a reducible, slightly-painful perineal swelling during the adjuvant therapy. He had had difficulties in sitting, walking and voiding due to increased perineal bulging during the previous two months. Physical examinations revealed an adult-fist-sized reducible mass (Fig. 1). Abdominopelvic computed tomography showed small bowel loops protruding into the pelvic cavity (Fig. 2).

After completion of the adjuvant therapy, we decided to repair 


\section{Coloproctology of Taek-Gu Lee and Sang-Jeon Lee}

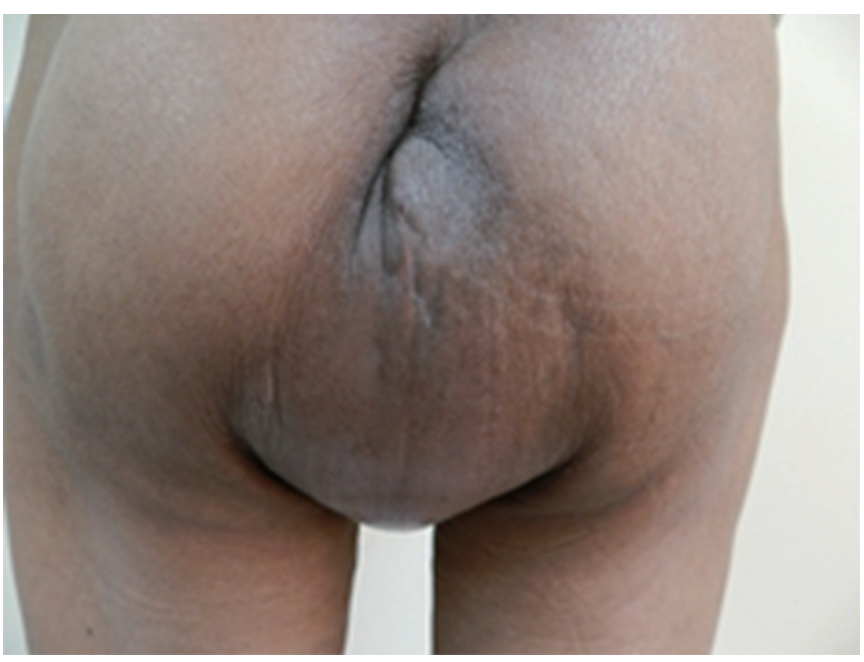

Fig. 1. Physical examination revealed adult-fist-sized reducible mass.

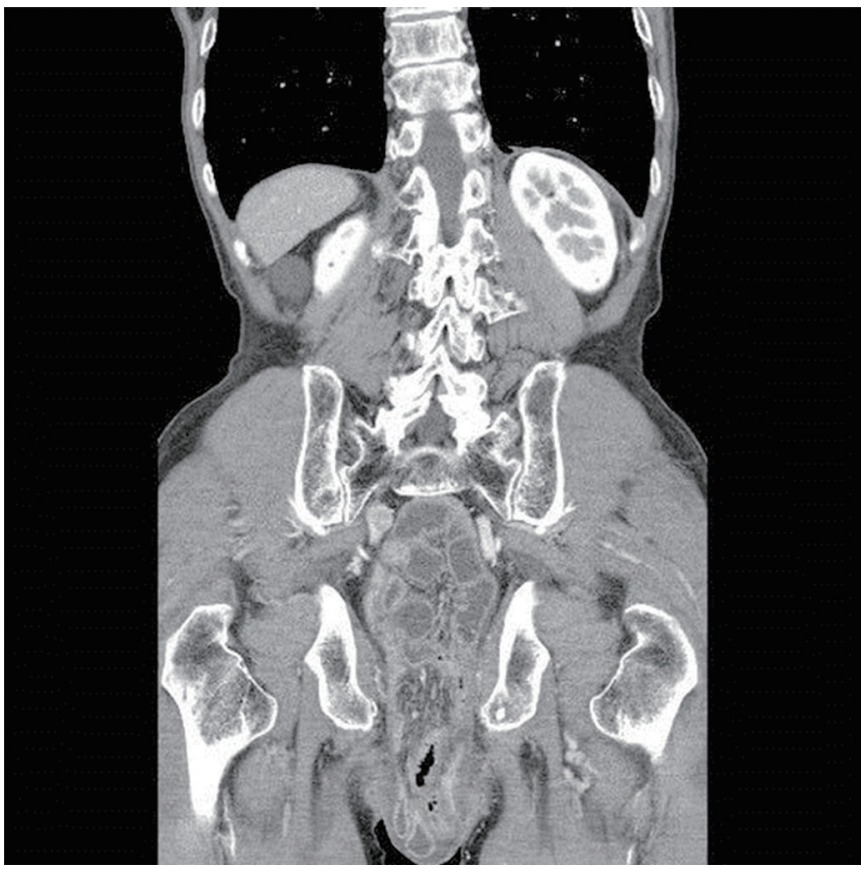

Fig. 2. Abdominopelvic computed tomography scan showed small bowel loops protruding into the pelvic cavity.

the hernia via a perineal approach. The patient was placed in the jackknife position under general anesthesia. Skin was incised along the previous incision. The hernia sac was dissected free and resected; then, it was reduced into the pelvic cavity (Fig. 3). The pelvic floor could not be primarily sutured due to the radicallydissected levator ani muscle. Thus, we applied Parietex ${ }^{\circledR}$ composite mesh (Covidien, Mansfield, MA, USA) for the reconstruction of the pelvic floor. The mesh was sutured with 2-0 Prolene in the anterior and the posterior directions. In the lateral direction, it was fixed to the ischial bony structures by using metallic, me-
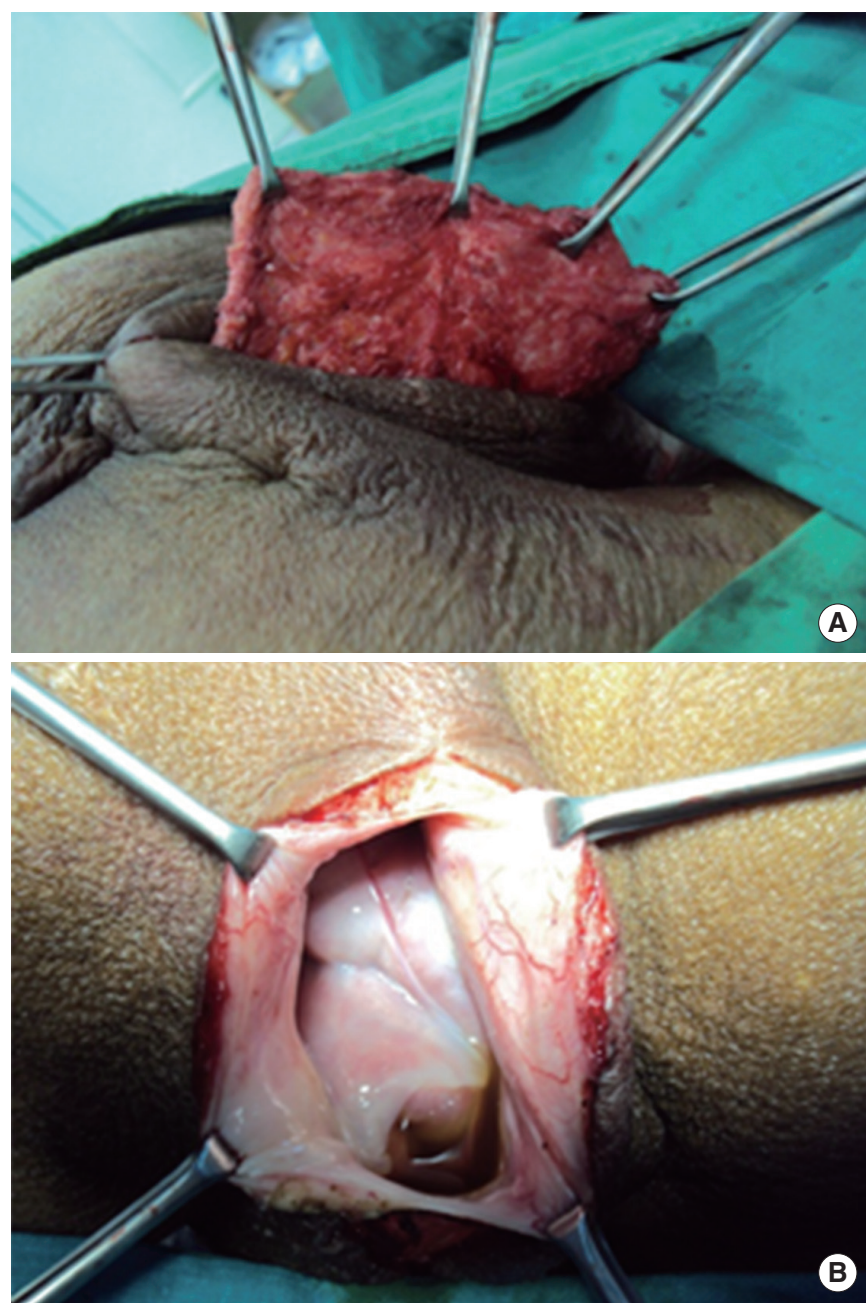

Fig. 3. The hernia sac was (A) dissected free and resected and (B) then reduced into the pelvic cavity.

chanical tacking devices (ProTack, Covidien) (Fig. 4). A JacksonPratt drain was inserted into the subcutaneous tissue.

The postoperative course was uneventful. He was discharged on the 9th postoperative day. During the 27-month follow-up, physical examinations and abdominopelvic computed tomography scans for postoperative surveillance showed no evidence of recurrence of the $\mathrm{PH}$ or cancer.

\section{DISCUSSION}

Different anatomic, technical, and clinical factors, including the larger size of the female pelvis, a previous hysterectomy, radiotherapy, coccygectomy, excessive length of small bowel mesentery, and perineal infection, may facilitate the formation of a $\mathrm{PH}$ after an APR [3]. Perineal wound complications, such as impaired perineal healing and infection, especially secondary to radiotherapy, seem to have an important etiological role [4]. Another important 


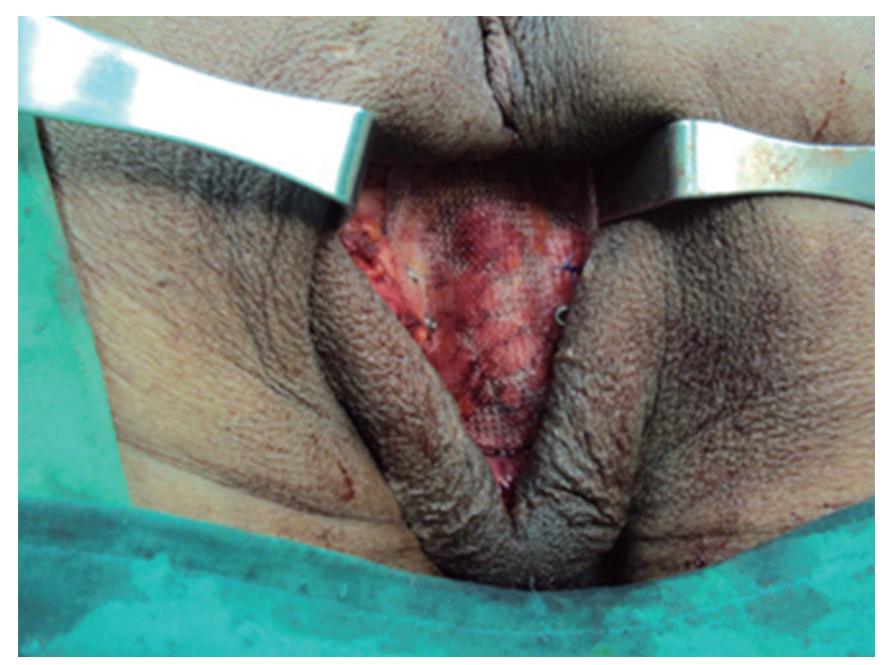

Fig. 4. Parietex ${ }^{\circledR}$ composite mesh (Covidien, Mansfield, MA, USA) was applied for the reconstruction of the pelvic floor. The mesh was sutured with 2-0 Prolene in the anterior and the posterior directions. In the lateral direction, it was fixed to the ischial bony structures by using metallic, mechanical tacking devices (ProTack, Covidien).

factor in the development of a PH after an APR is the extent of resection of the levator muscles. Although the extralevator APR improves oncological outcome, it increases the risks of perineal wound complications and probably the development of a $\mathrm{PH}$ [5]. One may expect a higher risk of $\mathrm{PH}$ in patients with the pelvic peritoneum left unsutured. However, the cause-effect relationship with peritoneal suturing is still controversial [6]. Higher risk may be observed after laparoscopic resections. The pelvic peritoneum is usually left open during the laparoscopic procedure. In addition, laparoscopic techniques can result in the formation of fewer adhesions in the abdominal cavity, so an increased potential exists for the small bowel loops to slide down toward the pelvis and to protrude into the perineal area more easily $[1,3]$.

Symptomatic hernias require treatment. The patients can be managed conservatively by using a T-bandage or a firm pair of underpants. A surgical approach to treatment can be via the perineum or the abdomen, with the latter approach being either open or laparoscopic. Alternatively, a combined abdominoperineal approach can be used. The main technical steps include identification of the defect, reduction of its contents, and repair of the defect. In late cases, the hernia sac must also be mobilized and excised. The abdominal approach allows the surgeon to have better exposure for dissecting the sac's contents and to confirm the absence of abdominal recurrence. However, the abdominal approach is much more invasive and is, accordingly, reserved for recurrent hernia patients. A transperineal approach causes less morbidity. Its main disadvantage is the limited exposure of the pelvis, making any potential tumor recurrence difficult to exclude. Mobilization of an adherent small bowel may also be difficult. Additionally, mesh fixation difficulties may lead to a high rate of hernia recurrence. However, the transperineal approach in the jackknife position can give sufficient exposure for repair, and the fewer adhesions after the initial laparoscopic procedure may not require dissection of the small bowel out of the pelvic cavity.

Surgical repair can be achieved by either primary suture or the use of a mesh or autologous tissue such as a muscular flap. The recurrence rate after primary closure (50\%) is higher than that after mesh repair (20\%), suggesting that mesh repair is the preferred technique [4]. The use of synthetic mesh, especially in a contaminated field, can carry a high risk of infection. Biological meshes may have advantages in complex repairs because of their nonsynthetic qualities. These meshes have an immunological intent and can be left in place in case of wound infection [2], but no data exist to support the use of a specific type of mesh. Recurrences after mesh repair are probably related to insufficient attachment to the pelvic side wall in the absence of a solid rim of the remaining levator complex. Ventrally, the mesh should be folded against the posterior vaginal wall or prostate with sufficient overlap to prevent sagging of the mesh. This will compensate for the inability to firmly suture the mesh ventrally [4]. An autogenous tissue flap reconstruction of the pelvic floor is rarely used for primary repair, but should be considered for patients with recurrent hernia, especially in the presence of irradiated tissue or infection $[3,7]$.

In conclusion, the perineal approach using mesh for the reconstruction of the pelvic floor is less invasive and more effective. We suggest that this technique should probably be the first choice for managing an uncomplicated PH after a laparoscopic APR.

\section{CONFLICT OF INTEREST}

No potential conflict of interest relevant to this article was reported.

\section{REFERENCES}

1. Akatsu T, Murai S, Kamiya S, Kojima K, Mizuhashi Y, Hasegawa $\mathrm{H}$, et al. Perineal hernia as a rare complication after laparoscopic abdominoperineal resection: report of a case. Surg Today 2009;39: 340-3.

2. Svane M, Bulut O. Perineal hernia after laparoscopic abdominoperineal resection--reconstruction of the pelvic floor with a biological mesh (Permacol ${ }^{\mathrm{TM}}$ ). Int J Colorectal Dis 2012;27:543-4.

3. de Campos FG, Habr-Gama A, Araujo SE, Sousa AH Jr, Nahas CR, Lupinacci RM, et al. Incidence and management of perineal hernia after laparoscopic proctectomy. Surg Laparosc Endosc Percutan Tech 2005;15:366-70.

4. Mjoli M, Sloothaak DA, Buskens CJ, Bemelman WA, Tanis PJ. Perineal hernia repair after abdominoperineal resection: a pooled analysis. Colorectal Dis 2012;14:e400-6.

5. Stelzner S, Koehler C, Stelzer J, Sims A, Witzigmann H. Extended abdominoperineal excision vs. standard abdominoperineal excision in rectal cancer: a systematic overview. Int J Colorectal Dis 
2011;26:1227-40.

6. So JB, Palmer MT, Shellito PC. Postoperative perineal hernia. Dis Colon Rectum 1997;40:954-7.
7. Brotschi E, Noe JM, Silen W. Perineal hernias after proctectomy: a new approach to repair. Am J Surg 1985;149:301-5. 